\title{
Psychosocial Contexts of Diabetes and Older Adulthood: Reciprocal Effects
}

CATHLEENM CONNELL, PhD

The University of Michigan

Ann Arbor, Michigan

The present study was conducted to assess the reciprocal effects between the psychosocial contexts of diabetes and older adulthood. Data were collected from 191 community-dwelling adults over the age of 60 with non-insulin-dependent diabetes mellitus. Results indicate that older adults with diabetes reported higher rates of selected chronic illnesses, lower self-rated physical health, and higher levels of depression than did comparison samples of older adults without diabetes. Compared with younger adults with NIDDM, the present sample of older adults perceived fewer impacts of diabetes, including fewer symptoms of poor metabolic control, less emotional impact, fewer barriers to adherence, and less complex regimens. Overall levels of social support and. regimen adherence were high. Older adults in this sample reported wanting minimal help from their family and friends with self-management activities and receiving more help than desired with following a meal plan and taking medications. Implications of the unique context of older adulthood for diabetes self-management are discussed.
Two factors have accelerated the increased attention to diabetes among older adults: the prevalence of diabetes increases with age, and more people are living longer. ${ }^{1}$ The unique psychosocial and physiological context of older adulthood has implications for diabetes treatment and management. A recent issue of Diabetes Care was devoted exclusively to diabetes mellitus in elderly people and covered such topics as the role of the diabetologist, geriatrician, and diabetes educator in the care of older adults with diabetes; complications of diabetes and issues related to comorbidity; and treatment options for older aduilts. ${ }^{2}$ To date, however, few empirical studies of individuals with diabetes have included older adults. Concomitantly, the gerontological literature has paid limited attention to the effect of diabetes on physical and mental health outcomes, despite the prevalence of the disease.

The present study was conducted to assess the reciprocal effects between the psychosocial contexts of diabetes and older adulthood. The first specific aim of the present study was to compare several psychosocial dimensions between representative samples of older adults and a sample of older adults with diabetes. The second specific aim of the present study was to assess several dimensions of the psychosocial context of diabetes between representative samples of both middle-aged and older adults with diabetes. The third specific aim was to report selected findings of a comprehensive psychosocial assessment of diabetes used with older adults with non-insulin-dependent diabetes mellitus (NIDDM). Topics to be addressed include overall understanding of diabetes and knowledge of complications, symptoms of poor control, regimen characteristics and adherence, social support related to diabetes, and the impact of diabetes on social activities and emotional well-being. Results that focus on predictors of adherence, depression, metabolic control, ${ }^{3}$ and gender differences in the impact of social support on diabetes outcomes ${ }^{4}$ have been reported elsewhere. (Although care was taken to select appropriate secondary data sources for contrast, caution is advised in interpreting results due to unique characteristics of each sample.)

Dr Connell is an AssistantProfessor, Department of Health Behavior and Health Education, School of Public Health, University of Michigan.

Funding for this research was provided in part by NIH grants AM 20579, HL07456, and 00030.

Reprint requests to Cathleen $\mathrm{M}$ Connell, $\mathrm{PhD}$, Department of Health Behavior and Health Education, School of Public Health, The University of Michigan, 1420 Washington Heights, Ann Arbor, MI 48109-2029. 


\section{Methods}

Comparison Samples of Older Adults To compare the present sample with representative samples on overall health status, selected chronic conditions, social support, and depression, several comparison samples were used. National norms for self-rated physical health were drawn from the National Health Interview Survey (NHIS), an ongoing, cross-sectional, nationwide survey conducted by household interview. ${ }^{5}$ A representative sample of 5,982 individuals over the age of 65 responded to the question, "Overall, how would you rate your physical health?" Response choices included poor, fair, good, very good, and excellent. For this comparative sample, $59.1 \%$ are women, $90.4 \%$ are white, $54.7 \%$ are married, and $46.7 \%$ have completed at least a high school education

For estimating selected chronic conditions in the noninstitutionalized population of the United States, national estimates for the years 1983 to 1985 were drawn from data provided by the National Center for Health Statistics. ${ }^{6}$ The data were collected as part of the NHIS. Estimates are provided for a sample of 26,456 individuals over the age of 65 , the group most appropriate for comparison with older adults with diabetes (who were all over the age of 59). For this comparative sample, $51.8 \%$ are women and $85.5 \%$ are white.

To compare the present sample with national norms for coexisting chronic illnesses, data were drawn from the Supplement on Aging. ${ }^{7}$ This supplement accompanied the 1984 NHIS and is based on a national probability sample of 16,148 completed interviews of noninstitutional adults over the age of 5.5. Simple counts of chronic illnesses/conditions are generated for each participant who responds "yes" to the question, "Have you experienced this condition in the past year/ now/ever?" The majority of this sample was female $(56.5 \%)$ and white $(90.1 \%)$.

Social support among the sample of older adults with diabetes was compared with data reported in an earlier validation study of the Social Provisions Scale with a sample of 494 community-dwelling older adults. ${ }^{8}$ Mean age for this sample was 73 years, $66 \%$ were women, $53 \%$ were married, $84 \%$ were white, and average years of education was 10 .

Depression scores were compared with data reported from a community study of older adult's scores on the Zung SelfRating Depression Scale. ${ }^{9}$ The 463 men and women between the ages of 60 and 79 in their study were from the same metropolitan area as the sample of older adults with diabetes, and were recruited in a similar fashion. Average level of educational attainment was 12.5 years.

\section{Comparison Samples of Adults With Diabetes}

Volunteers were recruited from the St Louis metropolitan area through press releases in the local newspaper and on the radio; presentations by research staff at diabetes support group meetings; and by notices posted at local retirement homes, housing complexes for older adults, and health clinics.

A total of 191 community-dwelling adults over the age of 60 with NIDDM participated in the study ( $\bar{X}$ age $=70.3$, SD $=6.7$ ). Sixty percent of the sample was female, $14 \%$ black, $53 \%$ married, and $56 \%$ completed at least high school. On average, the sample has lived with diabetes for almost 14 years. In-home interviews were arranged with each study participant.

The Diabetes Care Profile (DCP) was developed at the Michigan Diabetes Research and Training Center ${ }^{10}$ and was included in a community-based assessment of 261 Michigan residents with diabetes. This Michigan-based sample included 28 individuals classified as having insulin-dependent diabetes mellitus (IDDM) and 233 individuals classified as having NIDDM. Two subsets were drawn from the sample of 233 individuals with NIDDM and were used for comparison with the St Louis sample of older adults: (a) 162 individuals 60 years of age or older, and (b) 71 individuals less than 60 years of age. Mean age of the older adult sample $(>60)$ was 69.6 years $(\mathrm{SD}=6.4)$, the majority were female $(63.2 \%)$ and married $(61.9 \%)$, and $58.7 \%$ had completed at least high school. Mean age of the second comparison sample $(n=71)$ was $51.7(\mathrm{SD}=7.2)$, the majority were female $(64.8 \%)$ and married $(76.1 \%)$, and $74.6 \%$ had completed at least high school.

\section{-Measures The following measures were used:}

Number of Chronic Illnesses This value refers to the total number of chronic illnesses that a participant reported. Possible scores ranged from 0 to 19 . Chronic illnesses included high blood pressure, low blood pressure, heart disease, arrhythmia, heart bypass, pacemaker, angina, heart attack, stroke, arthritis, osteoporosis, bronchitis, emphysema, asthma, cancer, Parkinson's disease, epilepsy, hypothyroid condition, and hyperthyroid condition.

Depression The 20-item Zung Depression Scale ${ }^{11}$ was included because of its reliability and validity and previous use with older adults. High scores indicate higher levels of depression. Raw scores were converted to index scores, according to established procedure." Chronbach's alpha for the scale was .77 .

Social Support The 24-item Social Provisions Scale, ${ }^{12}$ designed for use with older adults, was chosen to assess the perceived availability of support. High scores for an overall summary score (SPS overall) indicate higher levels of social support. Chronbach's alpha for the scale was .91 .

Diabetes Care Profile This multimeasure assessment of an individual's adaptation to diabetes includes nine subscales.

Perceived Risk of Complications is a 5-item subscale that assesses the extent to which an individual feels that optimal diabetes self-management will prevent eye, kidney, and foot problems, hardening of the arteries, and heart disease. High scorers perceive that optimal self-care will prevent complications of diabetes. This variable will be referred to as risk of complications.

Perceived Symptoms of Poor Control is an 18-item subscale that indicates the frequency with which an individual reported symptoms of poor metabolic control (eg, reactions due to high or low blood glucose levels in the past month, the number of diabetes-related hospitalizations in the past year, and the frequency with which diabetes prevented the individual from conducting his or her normal activities). High scorers report more perceived control problems than low 
Table 1. Comparison of the Health Status and Psychosocial Context of the General Population of Older Adults With Older Adults With Diabetes

\begin{tabular}{|c|c|c|}
\hline & General & Diabetes \\
\hline \multicolumn{3}{|l|}{ Self-rated phȳyical health ${ }^{\mathbf{a}}$} \\
\hline Poor & $11 . \overline{5}$ & 13.6 \\
\hline Faiü & 21.4 & 30.4 \\
\hline Good & 31.9 & 38.7 \\
\hline Very good & 19.2 & 11.5 \\
\hline Excellent & -15.6 & 5.8 \\
\hline \multicolumn{3}{|c|}{ Prevalence of selected chronic conditions ${ }^{b}$} \\
\hline Arthritis & 48.4 & 67.2 \\
\hline Asthma & 3.6 & 4.7 \\
\hline Bronchitis & 6.0 & 21.4 \\
\hline Cataracts & 34.9 & 16.9 \\
\hline Emphysema & 4.1 & 5.2 \\
\hline Heart disease & 14.0 & 27.1 \\
\hline Hypertension & 41.8 & 53.6 \\
\hline Retinopathy & 1.6 & 14.6 \\
\hline Number of chronic illnesses ${ }^{c}$ & $2.7(1.6)$ & $3.5(2.1)$ \\
\hline \multicolumn{3}{|l|}{ Depression } \\
\hline Total sample & $43.7(8.0)$ & $49.7(8.6)$ \\
\hline Men & $42.6(8.0)$ & $44.5(7.1)$ \\
\hline Women & $44.3(8.0)$ & $53.5(8.3)$ \\
\hline Social support ${ }^{\mathrm{e}}$ & $77.4(13.8)$ & $76.2(9.3)$ \\
\hline \multicolumn{3}{|c|}{ 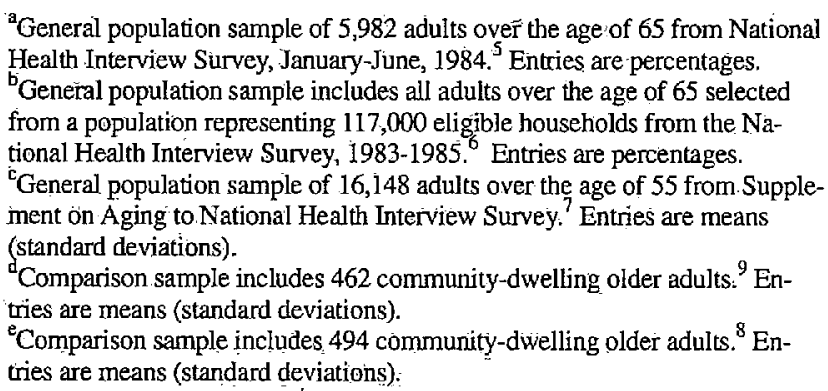 } \\
\hline
\end{tabular}

scorers. This variable will be referred to as control problems.

Regimen Adherence is a 23-item subscale that assesses the extent to which an individual adheres to the regimen recommended by his or her health care professional. Components of the regimen include diet, exercise, oral medication/insulin, glucose monitoring, and foot care. High scorers report that they adhere more closely to their regimen than do low scorers. This variable will be referred to as adherence.

Diabetes-Specific Social Support is a 10-item subscale that assesses the extent to which individuals perceive that their social networks provide tangible and emotional support related to the management of their diabetes. Items assess whether friends and family help with taking medication, adherence to the meal plan, foot care, getting enough physical activity, glucose testing, offering acceptance, and providing encouragement. High scorers perceive more support to be available than do low scorers. This variable will be referred to as social support.

Social Impact of Diabetes is an 8-item subscale that assesses the extent to which an individual feels that diabetes interferes with their social activities, including traveling, being active, eating. preferred foods, having good relationships with others, and meeting their work responsibilities.
High scorers perceive that diabetes has a greater sôciâl impact on their lives than low scorers. This variable will be referred to as social impact.

Emotional Impact of Diabetes is a 10 -item subscale that assesses the extent to which an individual perceives that diabetes has had an impact on his or her overall life satisfaction, self-esteem, and happiness. High scorers perceive that diabetes has had a greater emotional impact on their lives than do low. scorers. This variable will be referred to as emotional impact.

Benefits of Adherence is a 6-item subscale that assesses the extent to which individuals perceive that adherence will help them to control their diabetes, including testing for glucose and ketones and following a meal plan. High scorers perceive more benefits to regimen adherence than do low scorers. This variable will be referred to as benefits.

Barriers to Adherence is a 14-item subscale that measures the extent to which barriers prevent or discourage individuals from adhering to their regimen, including its medication/insulin requirements, glucose monitoring, and exercise program. High scorers perceive more barriers than do low scorers. This variable will be referred to as barriers.

Regimen Complexity is a 23-item subscale that assesses the complexity of the regimen that has been recommended by an individual's physician or health care professional. Regimen components include diet, exercise, meal schedule, use of pills or insulin, and foot care. High scorers perceive that their regimen is more complex than do low scorers. This variable will be referred to as regimen complexity.

To address the third purpose of the present study, responses to individual items from the following subscales are reported: (a) Regimen Adherence, (b) Diabetes-Specific Social Support, (c) Social Impact of Diabetes, and $(d)$ Emotional Impact of Diabetes. Additionally, items representing diabetes understanding, knowledge of complications, and symptoms of poor control are reported.

\section{Results}

Comparisons With Samples of Older Adults As shown in Table 1, the present sample of older adults with diabetes was compared with the previously described samples of older adults on various health status and psychosocial dimensions. Older individuals with diabetes reported poorer health than did the representative sample of older adults, $\chi^{2}$ $(4, N=191)=14.86, P<.01$. A number of chronic illnesses were reported significantly more frequently by older adults with diabetes than by a large representative sample of older adults without diabetes. These illnesses included arthritis, bronchitis, heart disease, hypertension, and retinopathy (all $P<.0001$ except for hypertension, which was $P<.02$ ). The diabetic older adults reported significantly fewer problems with cataracts $(z=-5.16, P<.0001)$ but did not differ from the general population in terms of asthma or emphysema. Using data from the NHIS Supplement on Aging, the average number of chronic conditions for older adults without diabetes was 2.7 , compared with 3.5 for the older adults with diabetes, $t(16,337)=-5.33, P<.0001$.

Scores indicate that approximately $53 \%$ of the present sample is within normal range in terms of their depression scores, indicating no psychopathology. The scores for over one fourth of the sample (27\%) indicate mild depression, 
Table 2. Diabetes Care Profile Scale Scores for Comparison Samples of Middle-Aged and Older Adults With NIDDM With the Present Sample of Older Adults With NIDDM

\begin{tabular}{|c|c|c|c|c|c|c|}
\hline \multirow{3}{*}{$\begin{array}{l}\text { DCP Subscale Scores } \\
\text { Risk of complications }\end{array}$} & \multicolumn{4}{|c|}{ Comparison Samples } & \multirow{2}{*}{\multicolumn{2}{|c|}{$\begin{array}{l}\text { Present Sample } \\
\geq 60 \text { years } \\
(N=191)\end{array}$}} \\
\hline & \multicolumn{2}{|c|}{$\begin{array}{c}<60 \text { years } \\
(N=71)\end{array}$} & \multicolumn{2}{|c|}{$\begin{array}{l}\geq \mathbf{6 0} \text { years } \\
(\mathbf{N}=162)\end{array}$} & & \\
\hline & 521.0 & (93.6) & 504.7 & (98.2) & 487.4 & $(83.0)$ \\
\hline Control problems & 540.5 & $(117.7)$ & 493.3 & $(102.9)$ & 504.1 & $(109.2)$ \\
\hline Adherence & 481.5 & (95.8) & 512.4 & (99.9) & 503.5 & $(105.3)$ \\
\hline Social support & 478.5 & $(99.4)$ & 501.9 & $(97.7)$ & 471.2 & $(87.0)$ \\
\hline Social impact & 494.8 & $(94.1)$ & 488.4 & (95.4) & 517.5 & $(82.6)$ \\
\hline Emotional impact & 512.2 & $(114.5)$ & 488.6 & $(102.0)$ & 488.9 & $(96.4)$ \\
\hline Benefits & 504.7 & $(98.7)$ & 502.2 & (113.9) & 480.6 & $(100.1)$ \\
\hline Barriers & 525.2 & $(110.8)$ & 483.5 & (94.2) & 473.2 & $(66.4)$ \\
\hline Regimen complexity & 512.7 & $(92.8)$ & 495.2 & $(100.2)$ & 540.1 & $(106.3)$ \\
\hline
\end{tabular}

Note: Entries are means with standard deviations in parentheses. The higher the score, the greater the dimension. Scores are standardized; $\overline{\mathrm{X}}=500$, $\mathrm{SD}=100$.

scores for $15 \%$ of the sample indicate moderate depression, and scores for $5 \%$ indicate severe depression. The mean Self-Rating Depression Scale (SDS) score for the older adults with diabetes was 49.7 (SD $=8.6$ ), compared with 43.7 ( $\mathrm{SD}=8.0$ ) for a sample of 463 community-dwelling older adults, ${ }^{9}$ indicating significantly higher levels of depression among the older adults with diabetes $t(649)=-7.8$, $P<.0001$. Although the women in the community sample. were slightly more depressed than were the men (44.3 versus. 42.6), the older women with diabetes were significantly more depressed than the older men with diabetes (53.5 versus 44.5) $t(187)=-6.3, P<.0001$.

In terms of the perceived availability of social support, the sample of older adults with diabetes had scores not significantly different from those of a comparative sample of older: community-dwelling adults. For the overall score of the Social Provisions Scale, the two samples perform almost identically in terms of mean scores and standard deviations. Overall, the present sample perceived that an adequate amount of support is available to them if needed.

Comparisons With Samples of Adults With Diabetes As shown in Table 2, subscale scores of the Diabetes Care Profile were compared between the St Louis sample of older adults with diabetes and the two Michigan samples. Scores for three subscales (Regimen Complexity, Social Support, and Social Impact) are different between the St Louis sample of older adults with diabetes and the Michigan sample of older adults. The present sample reports lower levels of diabetes-specific social support and greater social impact of the disease. Additionally, the present sample perceives that their diabetes regimen is more complex (includes more components) than does the comparison sample of older adults. No significant differences between the present and comparison sample of older adults were discovered for the perceived risk of complications, control problems, adherence, emotional impact of diabetes, and benefits of and barriers to adherence.

Compared with the younger Michigan sample, both samples of older adults reported fewer control problems, higher levels of adherence, less emotional impact of diabetes, and fewer barriers to regimen adherence. Compared with both
Michigan samples, the St Louis sample of older adults perceived a greater social impact to their illness, fewer benefits, and greater regimen complexity.

\section{Diabetes Care Profile-Item-Level Analyses Item-level} analyses revealed the following:

Understanding of Diabetes and Knowledge of Complications One third of the sample $(32 \%)$ rated their overall understanding of diabetes as "very good" or "excellent"; one third as "good" (37\%); and one third as "poor" or "fair" (31\%). The vast majority of the sample was aware of the relationship between diabetes care and the delay or prevention of complications, including eye problems (mentioned by $93 \%$ of the sample as preventable with proper diabetes care), foot problems (92\%), kidney problems (89\%), heart disease $(8.1 \%)$, and hardening of the arteries $(71 \%)$.

Symptoms of Poor Control Almost one fourth of the sample $(23 \%)$ reported having experienced symptoms of high blood glucose levels (ie, thirst, decreased appetite, fatigue) more than three times in the past month. Fewer participants $(12 \%)$ reported having experienced symptoms of low blood glucose levels during that same time period (ie, sweating, anxiety, trembling). Over half of the sample reported having no symptoms of low blood glucose levels (58.9\%) or high blood glucose levels (56.3\%) in the past month.

Regimen Characteristics and Adherence The vast majority of older adults in the St Louis sample (91\%) were told by their health care provider to follow a meal plan or diet. As can be seen in Table 3 , about three fourths of the sample $(74 \%)$ was advised to follow a schedule of meals and snacks. Of those advised to follow a schedule, over two thirds of the sample $(69 \%)$ did so on a regular basis (25\% responded "always" and $44 \%$ responded "usually"). Eleven percent never followed a meal plan or diet, even though it was recommended. Of the total sample, only one half followed a meal plan or diet.

Seventy percent of the sample was advised to weigh or measure their food, but only $21 \%$ did so on a regular basis ( $8 \%$ responded "always" and $13 \%$ responded "usually"). In 
Table 3. Number and Percentage of Older Aduilts for Whom Regimen Behaviors Have Been Prescribed and Number and Percentage Who Perform These Behaviors

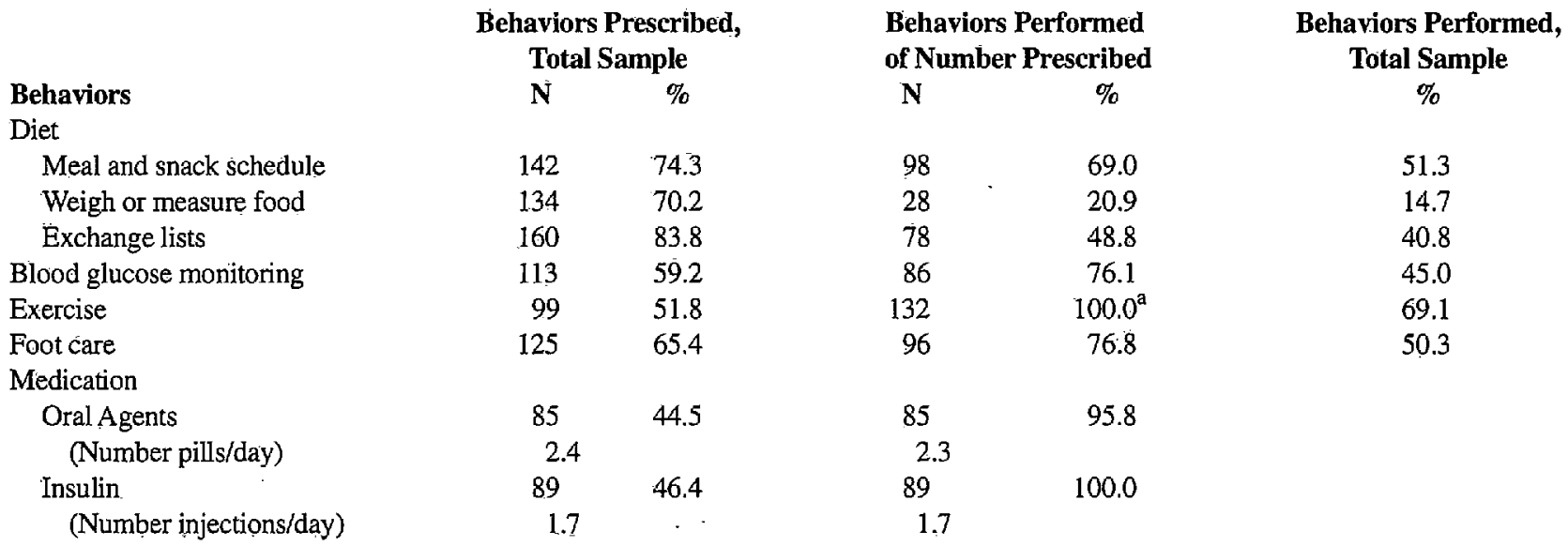

${ }^{\mathrm{a}} \bar{E}$ xercise has been prescribed for 99 participants ( $51.8 \%$ of the sample); 133 participants $(69.3 \%)$ report that they exercise.

fact, $40 \%$ of the sample reported that they never weighed or measured their food even though they had been instructed to do so. Of the total sample, only $15 \%$ weighed or measured their food.

Over three fourths of the sample $(84 \%)$ had been advised to use exchange lists to plan their meals. Of this group, almost half used exchange lists on a regular basis (26\% responded "always" and 23\% responded "usually"). Another one fourth of the sample reported that they never used exchange lists, even when advised to do so $(23 \%$ of the sample). Of the total sample of older adults, $40 \%$ used exchange lists:

Blood glucose testing was the most frequently recommended monitoring technique ( $59 \%$ of the sample were told to monitor their blood glucose levels), followed by checking body weight $(55 \%)$, and testing urine (23\%). Over three fourths of the sample reported that they usually monitor their blood glucose levels (about 50\% reported that they "always" monitored, 26\% responded "usually," $18 \%$ responded "sometimes," and 1\% "never"). (Seè Täble 3.)

As can be seen in Table 3, slightly over half of the sample was instructed by a health care professional to exercise (52\%); over two thirds of the sample (69\%) reported that they exercised. When asked about the type and frequency of exercise, $42 \%$ of the exercisers reported daily activity, $12 \%$ exercised five to six times a week, and $24 \%$ exercised three to four times a week. The activity that most exercisers èngaged in was walking ( $46 \%$ of the exercisers); $17 \%$ used a stationary bike.

Two thirds of the sample $(65 \%)$ had been told to take special care of their feet. Of this group, over three fourths checked their feet every day (77\%) (See Table 2.) An additional $13 \%$ checked their feet at least once a week (13\%), and $7 \%$ never checked their feèt (7\%). Overall, one hălf of the total sample took special care of their feet.

Almost equal percentages of the sample took pills to regulate their diabetes $(45 \%)$ as took insulin (47\%). The average number of pills recommended per day was 2.4 ( $\mathrm{SD}=1.6$ ). Self-reported adherence was exceptionally high, with the average number of pills taken per day reported to be 2.3 (SD
$=1.5$ ). Similarly, adherence in terms of insulin administration was exceptionally high. The number of insulin injections recommended per day was $1.7(\mathrm{SD}=.69)$; the average number administered per day was reported to be identical $(\overline{\mathrm{X}}=1.7 ; \mathrm{SD}=.69) \cdot($ See Table 3.$)$

Diabetes-Specific Social Support Less than one third of the sample reported wanting help in following a meal plan (29\%), getting enough physical' activity (27\%), taking special care of their feet $(13 \%)$, taking medication $(12 \%)$, or testing for glucose (11\%). Almost twice as many older adults reported receiving help with following a meal plan and taking their medication than wanted help with these regimen activities ( $52 \%$ received help with a meal plan, and $21 \%$ with taking medication). Nearly identical percentages received help with foot care, physical activity, and glucose testing as reported wanting help with these regimen activities.

The majority of the sample perceived that their family and friends accepted them and their diabetes (mentioned by $92 \%$ of the sample), listened to them about their diabetes (69\%), and encouraged and reassured them about their diabetes (55\%). Very few older adults reported that their family and friends made them feel uncomfortable because of their diabetes $(8 \%)$ or discouraged or upset them about their diabetes $(7 \%)$.

Social Impact of Diabetes For the majority of older adults in the sample, diabetes was perceived as preventing individuals from "being as active as I want" (reported by $81 \%$ of the sample). Other impacts of diabetes on social activities included: (a) "paying for my diabetes is a problem" (mentioned by $32 \%$ of the sample), $(b)$ "going out or traveling as much as I want" ( $31 \%)$, (c) "having good relationships with people," (21\%), (d) "having a schedule I like" (19\%), (e) "meeting responsibilities" (19\%), and $(f)$ "eating food that I like" $(10 \%)$. Overall, however, the majority of the sample reported that diabetes did not prevent them from engaging in their normal daily activities (78\%).

Emotional Impact of Diabetes Less than one third of the 
sample felt that having diabetes made their life very difficult (23\%), felt unhappy and depressed because of their diabetes $(18 \%)$, or felt inferior to others because of their diabetes $(5 \%)$. The majority of the sample reported having no difficulty taking care of their diabetes (63\%) and not being afraid of their diabetes $(65 \%)$.

\section{Discussion}

Although care should be taken in contrasting the present sample with secondary sources, older adults with diabetes reported higher rates of selected chronic illnesses and lower self-rated physical health than national samples of older adults. This finding can be explained, in part, by the fact that several chronic conditions (eg, high blood pressure, heart disease) are associated with diabetes, In addition to the $60 \%$ of the sample who reported that diabetes was their most significant health problem, another $10 \%$ reported that heart disease was, and almost $3 \%$ mentioned high blood pressure as most important. Of the remaining $27 \%$, most reported that their most significant health problem was some other complication or condition associated with diabetes: obesity, neuropathy, blindness, vision or vascular problems, and an amputation. Diabetes, then, may contribute to a broader context of declining physical health and chronic illness in older people.

In terms of the psychosocial context of living with a chronic illness, the older adụlts with diabetes did not perceive less available social support than did the older adults in a validation sample for the selected social support scale. Individual differences in the perception and use of support, however, should be expected. ${ }^{13}$ The present study suggests that not all older individuals with diabetes may need or desire a great deal of support.

Results from the present study indicate that older adults with diabetes, especially women, are more depressed than a comparison sample of older adults. Several gender differences in this sample may contribute to the high rates of depression among women. Compared with the men, the women were significantly older, reported more chronic illnesses and lower levels of perceived social support, and were more likely to rate their physical health as poor or fair (all $P<.05$ ).

\section{Comparisons With Other Samples of Adults With} Diabetes The St Louis sample and the Michigan sample of older adults with NIDDM are similar in terms of the psychosocial context thought to influence the management of diabetes. Thus, the St Louis sample can be considered representative of community-dwelling older adults with NIDDM, with minor exceptions. Specifically, the present sample of older adults reports less social support and a greater social impact of their illness. This finding may be explained, in part, by the fact that approximately one fourth of the present sample resided in congregate housing for older adults. Older adults may have chosen this type of living arrangement because their social support had diminished (ie, due to death of a spouse) or because of physical health limitations (ie, other chronic illnesses). Altematively, characteristics of this type of housing may limit access to supportive others, contributing to the perception that diabetes interferes with social activities and relationships. The present sample also reports that their regimen is more complex than the comparison sample of older adults, possibly reflecting the especially high levels of comorbidity among the congregate housing residents.

Overall, the age-based comparisons indicate that the older adults perceive fewer psychosocial impacts of diabetes than do the middle-aged adults. Specifically, the older adults report fewer symptoms of poor metabolic control, less emotional impact of diabetes, fewer barriers to adherence, and less complex regimens than do middle-aged adults with NIDDM. Similarly, older cancer patients have been reported to experience less psychosocial disruption from cancer than do younger individuals. ${ }^{14}$ The diminished psychosocial impact of diabetes among older adults may be due to a variety of factors: (a) most older adults live with several chronic illnesses, $(b)$ the perception that health limitations are an inevitable part of the aging process, (c) fewer competing demands from work and family obligations, or $(d)$ the perception among older adults that diabetes is not an especially serious chronic illness. For some older adults, the fact that diabetes is perceived to have a limited impact on their lives may reflect a realistic adaptation to their illness. For others, however, this perception may inhibit their efforts to engage in self-care behaviors to manage their illness. The betweengroup differences for the remaining subscales were not related to age: social impact of diabetes, social support, benefits of adherence, and regimen complexity.

\section{Role of Social Support in the Management of Diabetes} Previous research suggests that diabetes-specific social support is a stronger determinant of self-care behavior than general measures of overall perceived support.15 The assumption is usually made that the availability of support will increase regimen adherence and have a positive impact on diabetes management. Two types of diabetes-specific social support were assessed in this study: emotional and tangible support.

The sample perceives high levels of emotional support related to the management of diabetes - encouragement, reassurance, and someone to listen. In terms of tangible support, the majority of older adults in this sample do not want a lot of help from their family and friends related to the management of their illness. Of those who indicate a preference for help, approximately equal numbers of older adults receive desired assistance with physical activity, foot care, and glucose testing. More older adults report receiving help with following a meal plan and taking medications, however, than report wanting help with these activities. Providing support that is not desired, even when offered with good intentions, may result in negative outcomes and may be perceived as nagging or as interference. ${ }^{16}$ Thus, the potential unintended negative consequences of an individual's support network on the management of a chronic illness should be anticipated. As suggested in previous research, the characteristics of and the relationship between potential support providers and recipients may determine whether support is perceived to be helpful or unhelpful..$^{17,18}$

Social and Emotional Impact of Diabetes Among Older Adults The treatment and management of diabetes may pose several barriers to an individual's social activities. For the majority of this sample of older adults, diabetes does not prevent involvement in normal daily routines, but does limit 
activity levels. This perception of limited activity may be due to the logistic and physiological demands of diabetes management - planning meals and medication, fatigue, and impairment in mobility caused by poor circulation or foot problems.

Diabetes does not have a strong impact on overall emotional health for the majority of older diabeties in this sample. As previously discussed, this may be due to the high levels of emotional support perceived to be available. Alternatively, individuals may perceive a limited need for emotional support related to their diabetes unless they experience symptoms or complications or perceive diabetes to be a serious illness. In this sample, infrequent physiological symptoms of high and low blood glucose levels and the high prevalence of other chronic conditions $(\bar{X}=3.5$ ) may limit the perceived severity of diabetes. For some older adults, diabetes may play a minor role in an older adult's perception of overall health status.

\section{Conceptualization and Measurement of Adherence} Among Older Adults Overall, the sample reports a fairly high level of understanding of diabetes and the vast majority indicate an awareness of the relationship between diabetes management and complications of the disease. Additionally, the sample reports very few symptoms of either high or low blood glucose levels. Knowledge and awareness of illness outcomes, however, have a tenuous relationship with longterm commitment to regimen adherence, especially when symptoms are infrequent. ${ }^{19}$

In the present study, individuals were first asked what regimen components their health care professional has recommended, followed by the extent to which these behaviors are performed. Regimen components include: following a meal plan, exercise, blood glucose monitoring, special foot care, and taking pills or insulin. As reported in several previous studies, self-reported adherence (behaviors performed/ behaviors prescribed) varies greatly, depending on the specific regimen component being described.20-22 For the St. Louis sample of older adults, adherence ranged from $21 \%$ for weighing and measuring food to almost $100 \%$ for taking pills and insulin. Research also documents the tendency for adherence to medical aspects of a regimen to exceed that for behavioral or life-style aspects, ${ }^{15,23,24}$ and this finding was confirmed with the present sample of older adults.

Self-reported adherence levels to broadly defined regimen components (eg, diet), however, may not accurately reflect the daily behavioral choices that influence diabetes management (timing and choice of meals, awareness of caloric and fat content). For example, although one half of the sample reports following a meal plan, only a few record calories, weigh or measure their food, or use exchange lists. Similarly, two thirds of the sample report that they perform blood glucose monitoring, but only one half of the sample own a blood glucose meter. Of this number, only a small minority record the results on a regular basis and even fewer use the results to alter their behavior or their treatment. Interestingly, two thirds of the sample report that they exercise, although only one half $(50 \%)$ have been instructed to do so for the management of their diabetes. Few older adults in the sample, however, exercise on a regular basis or at a level required for cardiovascular benefit. As previously mentioned, adherence to medical aspects of a regimen is exceptionally high. The vast majority of the sample reports taking their diabetes medication (pills or insulin) as prescribed. To increase adherence to behavioral regimen components, the multiple benefits of developing and maintaining.healthy lifestyles should be emphasized for older adults. For example, improved diet and exercise habits may result in weight loss, increased stamina and strength, regulation of appetite and sleep patterns, decreased blood pressure and cholesterol, and increased morale.

A limitation of the present study is its exclusive reliance on self-report measures of major study constructs. Of particular concern are self-reported measures of diabetes self-care behaviors that may overestimate adherence when compared with physiological data and reports from health care professionals and significant others. ${ }^{25}$ Data collection strategies in addition to self-report, however, are certainly recommended for research on older adults with diabetes; these include physician reports, observations of significant others, use of medical records, health diary booklets, periodic telephone calls, and qualitative methods.

Although the problems with defining and measuring adherence have been discussed at length in the diabetes literature, ${ }^{25}$ the potentially unique concerns for research involving older adults have not been addressed. Research on adherence usually assumes the following: (a) regimen components are known and understood by the individual with diabetes-the what, when, and how of the regimen; $(b)$ the individual with diabetes understands the prescribed regimen and its longterm behavioral implications (changes in diet, exercise) and intends to follow it; $(c)$ the prescribed regimen is appropriate to meet unique medical and psychosocial needs; $(d)$ the regimen can be recalled accurately; and $(e)$ increased adherence will result in better disease management.

Several of these assumptions related to the conceptualization of adherence may not be appropriate for research with older adults. Impaired cognitive functioning, illiteracy, and declining reading ability may limit a complete understanding of a complex regimen for some older adults. ${ }^{26}$ Because of the relatively recent emphasis on preventive and behavioral approaches to health care, some older adults may be more resistant to the nonmedical aspects of their regimen (ie, diet, exercise) than are younger adults. Additionally, health care professionals may place less emphasis on behavioral regimen components based on a stereotype of older adults as inflexible and unwilling to change existing habits. Many older adults encounter an increasing number of health-related problems that require medical attention (eg, systolic hypertension, urinary incontinence) and may necessitate changes in prescribed diabetes treatment. Finally, comorbidity may result in the perception that diabetes and adherence to a diabetes regimen may be perceived both by older adults and their health care professionals as of low priority compared with the management of other illnesses (eg, heart disease).

Most older adults in this sample received their diabetes regimen prescription when their disease was first diagnosed, which for most was over 10 years ago. Thus, recall errors may be significant, contributing to misperceptions of what the regimen entails. If the regimen is not fully understood, self-report adherence measures may result in misleading in- 
formation. (Perfect adherence to a regimen that differs significantly from the one prescribed may be less desirable in terms of diabetes management than low levels of adherence to a regimen that is remembered correctly.) Finally, the relationship between adherence and overall diabetes management may be unique for older adults, due in part to physiological and perceptual processes of aging, comorbidity, and polypharmacy. ${ }^{27}$

\section{Conclusions}

Findings from this study suggest that older adults perceive diabetes to have less impact on their lives and their overall health status than do younger adults. The variability among older adults, however, should not be underestimated. In this study, subtle differences in the psychosocial impact of diabetes between two independent samples of older adults with diabetes were discovered. Several determinants of the psychosocial impact of diabetes and regimen adherence may influence older adults to a greater extent than they do younger adults; these determinants include living arrangement, marital status, educational level, overall health status, perceived severity of the illness, cognitive status, reading ability, comorbidity and polypharmacy.

The author gratefully acknowledges Martha Storandt for her helpful comments on an earlier version of this manuscript, Cheryl Houston for her contribution to data collection and project management, Wayne $\mathrm{K}$. Davis and George Hess for their willingness to provide comparative data for this study, Edwin B. Fisher, Jr, for his support and encouragement of this project, and all of my colleagues at the Washington University and Michigan Diabetes Research and Training Centers.

\section{References}

1. Wingard DL, Sinsheimer P, Barett-Connor EL, McPhillips. JB. Community-based study of prevalence of NIDDM in older adults. Diabetes Care 1990;13(suppl 2):3-8.

2. Diabetes mellitus in elderly people. Special Issue. Diabetes Care 1990;13.

3. Kovar MG. Aging in the eighties, preliminary data from the supplement on aging to the national health interview survey: United States-January-June 1984. Hyattsville, Md: National Center for Health Statistics, 1986; DHHS publication no. (PHS)86-1250. (Advance Data from Vital and Health Statistics.)

4. Connell $\mathrm{CM}$, Storandt $\mathrm{M}$, Lichty $\mathrm{W}$. Impact of health belief and diabetes-specific psychosocial context variables on self-care behavior, metabolic control, and depression of older adults with diabetes. Behav Health Aging (in press).

5. Connell CM, Fisher EB $\mathrm{J}_{\mathrm{r}}$, Houston CA. The relationship between social support, morale, and diabetes outcomes among older men and women. J Aging Health (in press).

6. Collins JG. Prevalence of selected chronic conditions: United States-1983-1985. Hyattsville, Md: National Center for Health Statistics, 1988; DHHS publication no. (PHS)88-1250.
7. Fitti JE, Kovar MG. The supplement on aging to the 1984 national health interview survey. Hyattsville, Md: National Center for Health Statistics, 1987; DHHS publication no. (PHS)87-1323. (Vital and Health Statistics; series 1 ; no 21.)

8. Blieszner R, Mancini JA. The social provisions scale: assessing the functions of older adults' close relationships. Gerontologist 1985;25:120.

9. Berry $M M$, Storandt $M$, Coyne A. Age and sex difference in somatic complaints associated with depression. J Gerontol 1984;39:465-67.

10. Hess GE, Davis WK, VanHarrison R. A diabetes psychosocial profile. Diabetes Educ 1986;12:135-40.

11. Zung WWK. A self-rating depression scale. Arch Gen Psychiatry 1965;12:63-70.

12. Cutrona CE, Russell DW. The provisions of social relationships and adaptation to stress. In: Advances in personal relationships. Vol 1. Greenwich, Conn: JAI Press, 1987:37-67.

13. Connell CM, D'Augelli AR. The contribution of personality characteristics to the relationship between social support and perceived physical health. Health Psychol 1990;9:192-207.

14. Ganz PA, Schag CC, Heinrich RL. The psychosocial impact of cancer on the elderly: a comparison with younger patients. J Am Geriatr Soc 1985;33:429-35.

15. Wilson W, Ary DV, Biglan A, Glasgow RE, Toobert DJ, Campbell DR. Psychosocial predictors of self-care behaviors (compliance) and glycemic control in non-insulin-dependent diabetes mellitus. Diabetes Care 1986;9:614-22.

16. Schaefer LC, McCaul KD, Glasgow RE. Supportive and nonsupportive family behaviors: relationships of adherence and metabolic control in persons with type I diabetes. Diabetes Care 1986;9:179-85.

17. Shumaker SA, Brownell A. Toward a theory of social support: closing conceptual gaps. J Soc Issues 1984;40:11-36.

18. Wortman C. Social support and the cancer patient: conceptual and methodological issues. Cancer 1984;53(May 15 suppl):2339-60.

19. Janz N, Becker MH. The health belief model: a decade later, Health Educ Q 1974;11:1-47.

20. Glasgow RE, McCaul KD, Schafer L. Self-care behaviors and glycemic control of type I diabetes. J Chronic Dis 1987;40:399-412.

21. Orme CM, Binik YM. Consistency of adherence across regimen demands. Health Psychol 1989;8:27-43.

22. Schafer LC, Glasgow RE, McCaul KD, Drecher M. Adherence to IDDM regimens: relationship to psychosocial variables and metabolic control. Diabetes Care 1983;6:493-98.

23. Ary D, Toobert D, Wilson W, Glasgow RE. A patient perspective on factors contributing to nonadherence to diabetes mellitus. Diabetes Care 1986;9:168-72.

24. Glasgow RE, Toobert DJ, Riddle M, Donnolly J, Mitchell DL, Calder D. Diabetes-specific social learning variables and self-care behaviors among persons with type II diabetes. Health Psychol 1989;8:285-303.

25. Kurtz SMS. Adherence to diabetes regimens: empirical status and clinical applications. Diabetes Educ 1990;16:50-59.

26. Funnell MM. Role of the diabetes educator for older adults. Diabetes Care 1990;13(suppl 2):60-65.

27. Minaker KL. What diabetologists should know about elderly patients. Diabetes Care 1990;13(suppl 2):34-46. 